\title{
MR Visualization of Spinal Dural Arterio-Venous Fistula Using T2-Weighted 3D SPACE-A Spin-Echo Technique
}

\author{
Franz A. Fellner ${ }^{1}$, Christine M. Fellner ${ }^{1}$, Rene Chapot $^{2}$, Kaveh Akbari1 \\ ${ }^{1}$ Institute of Radiology, Kepler University Clinic, Medical Faculty of the Johannes Kepler University, Linz, Austria \\ ${ }^{2}$ Institute ofNeuroradiology, Alfried-Krupp-Krankenhaus, Essen, Germany \\ Email: franz.fellner@akh.linz.at
}

Received 12 March 2015; accepted 5 May 2015; published 6 May 2015

Copyright ( 2015 by authors and Scientific Research Publishing Inc.

This work is licensed under the Creative Commons Attribution International License (CC BY).

http://creativecommons.org/licenses/by/4.0/

(c) (i) Open Access

\section{Abstract}

Spinal dural arteriovenous fistulas (SDAVF) account for about $70 \%$ of all spinal AV shunts. We report on a 56-year-old male patient with progressive neurological symptoms. Pre- and post-contrast MR imaging of the spine, including contrast-enhanced MR angiography was done on a $1.5 \mathrm{~T}$ MR scanner. In addition a special (pre-contrast) 3D turbo-spin-echo sequence with variable flip angle evolution (SPACE) was performed providing a slice thickness of $0.9 \mathrm{~mm}$. T2-weighted images showed extensive widened and tortuous vascular structures with typical flow voids intradurally due to dilated pial veins. The origin of the spinal dural arteriovenous fistula was found to be the radiculomeningeal artery at level D10/11 on the left side, visualized by the T2-weighted 3D SPACE sequence and CE MRA. After imaging the patient was referred to an interventional neuroradiology department for therapy. With the detailed information from MR imaging concerning the fistula level the interventional radiologist was able to find the fistula immediately and consequently treated it successfully by endovascular therapy. Interpreting the MR images of this obvious case of a SDAVF, SPACE sequence after post processing with 3D MPR software directly led to the location of the AV shunt at D10/11 on the left side, better than 3D contrast-enhanced MR angiography did. Therefore, SPACE can be a useful adjunct in further evaluation of spinal dural arteriovenous fistula and especially in defining fistula level.

\section{Keywords}

Contrast Enhanced Magnetic Resonance Angiography (CE MRA), 3D Pulse Sequences, 3D SPACE, Spinal Dural Arterio-Venous Fistula (SDAVF) 


\section{Introduction}

Spinal arteriovenous malformations are commonly divided into four subtypes according to the Anson and Spetzler classification [1]:

- Type I: dural arteriovenous fistula (AVF), with single (IA) or multiple (IB) arterial feeders.

- Type II: intramedullary, glomus-type arteriovenous malformation (AVM).

- Type III: juvenile type AVM, with intramedullary, extramedullary and sometimes extradural components.

- Type IV: the perimedullary fistulous AVM, located intradural extramedullary.

About $70 \%$ of all spinal AV shunts are spinal dural arteriovenous fistulas (SDAVF). They are usually regarded as acquired lesions, although the exact etiology is not really known [2]. In contrast to cerebral dural arteriovenous fistulas, where an association with venous sinus thrombosis has been described, it seems to be unlikely that prothrombotic factors play a role in the pathogenesis of this lesion [3]. There is a significant gender predominance with men 4 to 5 times more often affected than women. Most often SDAVF are found in the midthoracicarea [4].

Venous congestion in SDAVF is due to arteriovenous shunting within the dura mater near to the spinal nerve root where the arterial blood from a radiculomeningeal artery drains into a radicular vein. Increase in spinal venous pressure diminishes the arteriovenous pressure gradient causing decreased drainage of normal spinal veins. Venous congestion leads to progressive myelopathy [2]. Subarachnoid hemorrhage is exceedingly rare in SDAVF [2], although an increased risk of bleeding in cervical DAVF has been reported, possibly due to intracranial drainage. Furthermore, venous varix is often found in cervical DAVF [5].

There are two treatment-options of SDAVF, surgical occlusion of the intrathecal vein receiving the shunt blood or endovascular therapy employing glue after superselective catheterization of the radiculomeningeal arterial feeder [2].

\section{Case Report}

We report on a 56-year-old male patient, who was referred to our institution with progressive neurological symptoms. The patient suffered from back pain and progredient paresthesias as well as motor weakness of both lower extremities. At that time the patient had already had symptoms for more than one year that gradually worsened over the past few months. He presented with diffuse back pain, paresthesias and paraparesis of the legs, so that he was forced to use crutches.

Pre- and post-contrast MR imaging of the spine, including MR angiography was done on a $1.5 \mathrm{~T}$ MR scanner (Magnetom Avanto, Siemens Erlangen, Germany). Standard two-dimensional imaging of the spine was applied with sagittal T2-weighted images including a T2*-weighted 3D GRE sequence (3D CISS = Constructive Interference in Steady State), sagittal T1-, axial T2-, as well as axial T1-weighted turbo spin-echo sequences before and after intravenous administration of gadolinium (Gadovist, Bayer, Germany). In addition a special 3D turbo-spin-echo sequence with variable flip angle evolution (SPACE $=$ Sampling Perfection with Application optimized Contrast using different flipe angle Evolutions) was performed (pre-contrast) providing the following sequence parameters: TR/TE 1500/244 ms; slice thickness 0.9 mm; matrix size $512 \times 512$; FOV $100 \mathrm{~mm}$. Furthermore, routine contrast enhanced MR angiography (CE MRA) was acquired as a 3D FLASH sequence (pre and post contrast enhancement) with image subtraction and subsequent multiplanar reformation (MPR) as well as maximum-intensity projection (MIP) evaluation.

MR tomography showed wide-stretching ill-delineated hyperintense signal alterations of the spinal cord on T2-weighted images ranging from the midthoracic region downwards to the conus medullaris representing typical myelon congestion as seen in spinal dural arteriovenous fistulas. Concomitant swelling of the spinal cord was noted. T2-weighted images additionally showed extensive widened and tortuous vascular structures with typical flow voids intradurally, somewhat accentuated on the dorsal parts of the myelon due to dilated pial veins (Figure 1).

The origin of the spinal dural arteriovenous fistula with a singular arterial feeder (Anson and Spetzler classification Type IA) was found to be the radiculomeningeal artery at level Th10/11 on the left side, visualized by the T2-weighted 3D SPACE sequence and CE MRA by means of MPR postprocessing (Figure 2).

Furthermore, multiplanar reformations in the coronal orientation visualized very well the dilated pial veins (Figure 3). Additionally, volume rendering of the 3D CE MRA enabled a clear three-dimensional visualization of the dural fistula (Figure 4).

The 3D turbo spin-echo sequence SPACE provided in comparison to 3D gradient-echo CISS a significantly improved overall image quality due to increased signal-to-noise-ratio. This was useful for better delineation of 

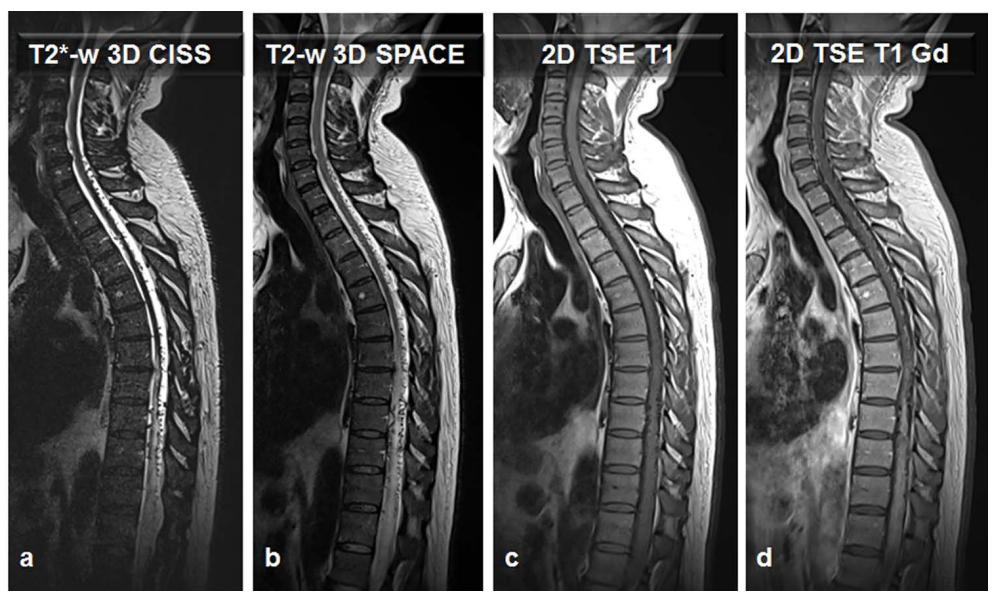

Figure 1. T2 ((a), (b))-weighted and Gd-enhanced T1-w images (d) show significantly engorged intradural veins from the lower cervical level down to the thoracolumbal level indicating that there is a spinal dural arterio-venous fistula. Furthermore, the caudal thoracic myelon is thickened and reveals increased signal intensity on T2-w as well on Gd-enhanced T1-w images due to venous congestion. The image quality of T2-w 3D spin-echo SPACE (b) is superior to that of T2*-w 3D GRE CISS (a) due to improved signal-to-noise ratio.
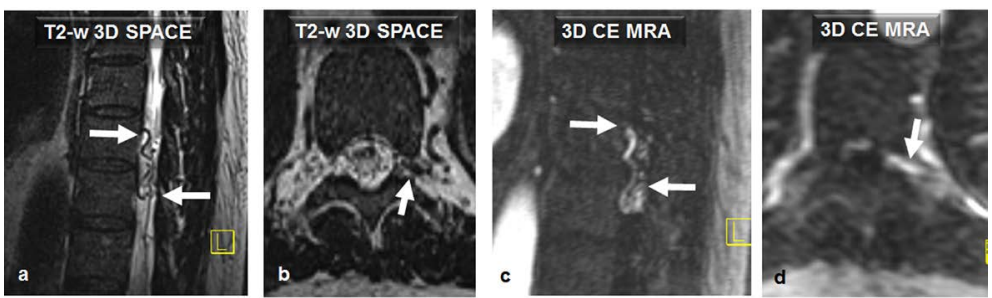

Figure 2. T2-w 3D SPACE ((a), (b)) and Gd-enhanced 3D CE MRA ((c), (d)) images show clearly the location of the spinal dural arterio-venous fistula (arrows) on sagittal images ((a), (b)) and transverse reconstructions ((b), (d)).
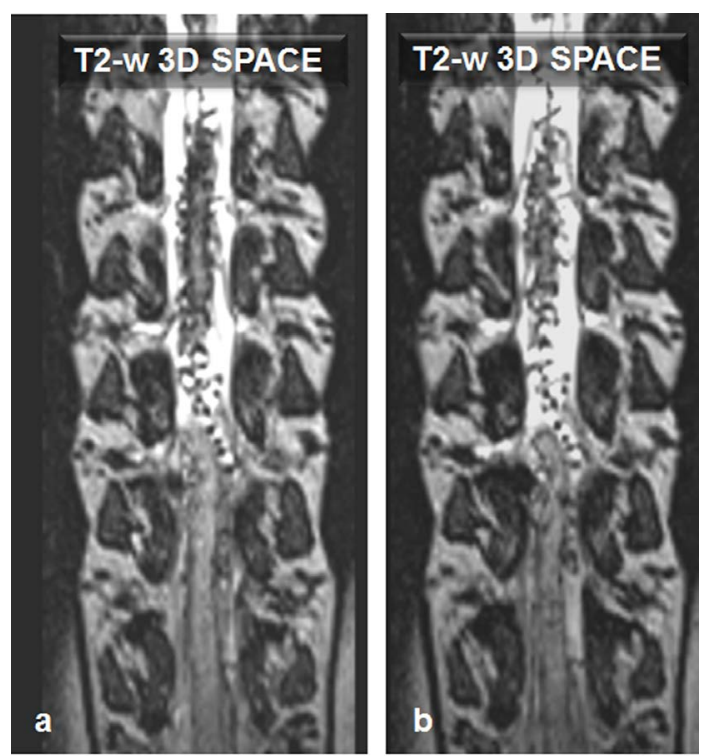

Figure 3. Coronal reformations of the T2-weighted 3D SPACE data set visualize very well the multiple dilated pial veins surrounding the spinal cord-the characteristic finding in patients with spinal dural arterio-venous fistula. 

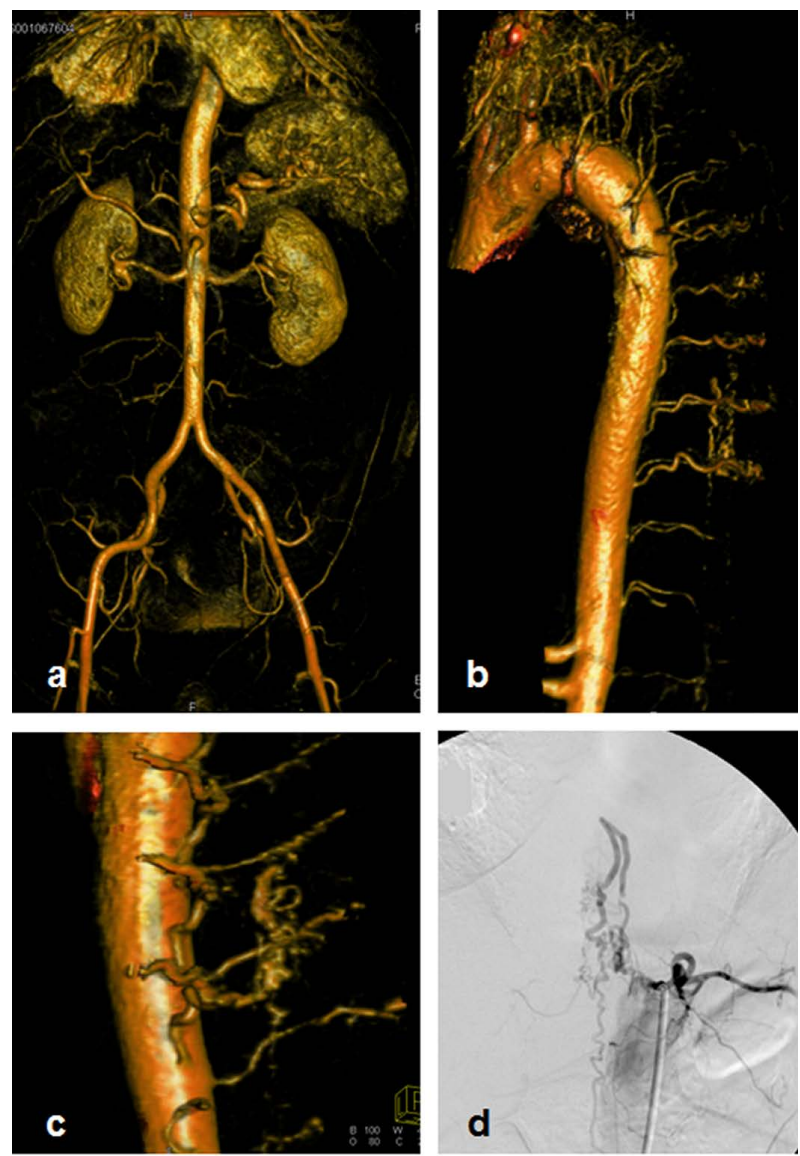

Figure 4. Postprocessing of 3D CE MRA using the volume rendering technique shows impressively normal vascularization of the lumbar aorta and run-off vessels (a) as well as the spinal dural arterio-venous fistula in the caudal thoracic level at Th 10/11 ((b), (c)). Selective catheter angiography of the fistula confirmed the MR diagnosis (d).

spinal structures, e.g. the vertebral bodies, and improved differentiation between the posterior longitudinal ligament and adjacent dilated veins.

After imaging the patient was referred to an interventional neuroradiology department for therapy. With the detailed information from MR imaging concerning the fistula level the interventional radiologist was able to find the fistula immediately (Figure 4(d)) and consequently treated it successfully by endovascular therapy.

\section{Discussion}

Diagnosis of spinal dural arteriovenous fistula often is delayed by months or even years [4]. Taking into account the rarity of the disease and the non-specific neurologic symptoms with an often slowly progressive course can explain that diagnostic imaging is often initiated too late. Possibly, this disease is underdiagnosed.

Once suspicion of vascular malformation has raised, MR imaging is considered to be the method of choice for primary visualization. MR imaging illustrates all pathology associated with SDAVF including spinal cord congestion with increase of T2 signal, swelling of the myelon and breakdown of blood-cord barrier as well as dilated perimedullary vessels [8]. As imaging features are rather characteristic in SDAVF, diagnosis is easily made with MR. However, an important aspect of MR screening for spinal dural arteriovenous fistula is detection of fistula level, which still remains challenging. Addition of MR angiography, especially 3D contrast-enhanced MR angiography, can improve determining of fistula level, with correct level (+/- one level) being identified in 73\% [9]. Nevertheless there are pitfalls and limitations of MR angiography, including inappropriate image coverage, subtraction misregistration artifacts, pseudodissection, phase artefacts, and insufficient spatial resoluton. Contrast-enhanced 3D MRA can indeed achieve an improved spatial resolution, but at the cost of temporal resolution. So it can be tricky to pinpoint the exact point of transition from arteries to veins as it may occur that 
by the time scan is completed, most of the first pass contrast is in the veins [10].

Considering these facts, spinal digital subtraction angiography (DSA) is currently regarded as the gold standard for the pretreatment detection and characterization of vascular malformations [11]. MR imaging combined with MR angiography plays a valuable role in confirming the diagnosis and targeting conventional catheter angiography. Predicting the level of fistulas by MR imaging substantially reduces the radiation load to the patient, total procedural time and volume of contrast agent associated with spinal catheter angiography [12]. Sharma et al assume at the present time that MRI/MRA is not able to replace catheter angiography as shown in their case report [10]. However, it must take into account that performance of an MR examination which is not state of the art may deliver false negative results.

This emphasizes the need for improving the robustness of MR imaging techniques in order to achieve higher sensitivity/specifity especially in defining the fistula level. 3D SPACE sequences may be a promising technique in this respect. SPACE is an acronym for Sampling Perfection with Application optimized Contrasts using different flip-angle Evolution. The sequence is a variant of the 3D turbo Spin-Echo (TSE) technique, designed as a single-slab 3D TSE with restore pulse and variable flip angle distribution which allows for extremely large turbo factors. In detail, the refocusing pulse train of the SPACE sequence consists of variable flip-angle pulses lower than $180^{\circ}$ resulting in radiofrequency energy deposition decrease. SPACE provides 3D thin-section isotropic data sets within an acceptable examination time. Values for SNR are comparable to 2D T2-weighted TSE sequences [6] [7]. SPACE combines highly resolved 3D datasets providing detailed anatomic information with high soft tissue contrast (T2-weighted, FLAIR contrast, IR T1 contrast). Up to now highly spatial and time-resolved T2-weighted 3D datasets have been based mainly on gradient-echo (GRE). Whereas GRE imaging techniques are not capable of providing a competitive T2-weighted contrast (in comparison to spin-echo techniques), 3D designed conventional spin-echo (SE) and turbo-spin-echo (TSE) schemes have not been applicable for a large field of view in clinical routine due to long acquisition times [7]. CISS (constructive interference in steadystate) representing a 3D T2-weighted gradient echo sequence on the other hand suffers from lack of tissue characterization and cerebrospinal fluid flow artifacts [6]. SPACE sequence can overcome these limitations.

Published literature on implementing SPACE in imaging of the spine is sparse. Meindl et al. [6] compared SPACE with conventional sequences for imaging of the cervical spine stating that sagittal 3D T2-weighted images enabled better delineation of nerve roots, neural foramina and intraforaminal structures compared to sagittal 2D T2-weighted images. Also Lichy et al. [7] emphasize the value of SPACE technique for imaging of complex anatomic regions, especially in cases of planning of spine surgery or resection of musculoskeletal tumors.

\section{Conclusion}

To our knowledge the presented case report is the first paper dealing with SPACE sequence in imaging of spinal dural arteriovenous fistula. Interpreting the MR images of this obvious case of a SDAVF, SPACE sequence after postprocessing with 3D MPR software directly led to the location of the AV shunt at Th10/11 on the left side, better than 3D contrast-enhanced MR angiography did. Therefore, SPACE can be a useful adjunct in further evaluation of spinal dural arteriovenous fistula and especially in defining fistula level.

\section{References}

[1] Anson, J.A. and Spetzler, R.F. (1992) Classification of Spinal Arteriovenous Malformations and Implications for Treatment. BNI Quarterly, 8, 2-8.

[2] Krings, T., Mull, M., Gilsbach, J.M. and Thron, A. (2005) Spinal Vascular Malformations. European Radiology, 15, 267-278. http://dx.doi.org/10.1007/s00330-004-2510-2

[3] Jellema, K., Tijssen, C.C., Fijnheer, R., de Groot, P.G., Koudstaal, P.J. and van Gijn, J. (2004) Spinal Dural Arteriovenous Fistulas Are Not Associated with Prothrombotic Factors. Stroke, 35, 2069-2071. http://dx.doi.org/10.1161/01.STR.0000135766.22285.dc

[4] Jellema, K., Canta, L.R., Tijssen, C.C., van Rooij, W.J., Koudstaal, P.J. and van Gijn, J. (2003) Spinal Dural Arteriovenous Fistulas: Clinical Features in 80 Patients. Journal of Neurology, Neurosurgery \& Psychiatry, 74, 1438-1440. http://dx.doi.org/10.1136/jnnp.74.10.1438

[5] Aviv, R.I., Shad, A., Tomlinson, G. Niemann, D., Teddy, P.J., Molyneux, A.J. and Byrne, J.V. (2004) Cervical Dural Arteriovenous Fistulae Manifesting as Subarachnoid Hemorrhage: Report of Two Cases and Literature Review. AJNRAmerican Journal of Neuroradiology, 25, 854-858.

[6] Meindl, T., Wirth, S, Weckbach, S., Dietrich, O., Reiser, M. and Schoenberg, S.O. (2009) Magnetic Resonance Imaging of the Cervical Spine: Comparison of 2D T2-Weighted Turbo Spin Echo, 2D T2*-Weighted Gradient-Recalled 
Echo and 3D T2-Weighted Variable Flip-Angle Turbo Spin Echo Sequences. European Radiology, 19, 713-721. http://dx.doi.org/10.1007/s00330-008-1175-7

[7] Lichy, M.P., Wietek, B.M., Mugler, J.P. $3^{\text {rd }}$, Horger, W., Menzel, M.I., Anastasiadis, A., Siegmann, K., Niemeyer, T., Königsrainer, A., Kiefer, B., Schick, F., Claussen, C.D. and Schlemmer, H.P. (2005) Magnetic Resonance Imaging of the Body Trunk Using a Single-Slab, 3-Dimensional, T2-weighted Turbo-Spin-Echo Sequence With High Sampling Efficiency (SPACE) for High Spatial Resolution Imaging: Initial Clinical Experiences. Investigative Radiology, 40, 754-760. http://dx.doi.org/10.1097/01.rli.0000185880.92346.9e

[8] Koch, C. (2006) Spinal Dural Arteriovenous Fistula. Current Opinion in Neurology, 9, 69-75. http://dx.doi.org/10.1097/01.wco.0000200547.22292.11

[9] Saraf-Lavi, E., Bowen, B.C., Quencer, R.M., Sklar, E.M.L., Holz, A., Falcone, S., Latchaw, R.E., Duncan, R. and Wakhloo, A. (2002) Detection of Spinal Dural Arteriovenous Fistulae with MR Imaging and Contrast-Enhanced MR Angiography: Sensitivity, Specificity, and Prediction of Vertebral Level. AJNR American Journal of Neuroradiology, 23, 858-867.

[10] Sharma, A.K. and Westesson, P.L. (2008) Preopertaive Evolution of Spinal Vascular Malformation by MR Angiography: How Reliable Is the Technique: Case Report and Review of Literature. Clinical Neurology and Neurosurgery, 110, 521-524. http://dx.doi.org/10.1016/j.clineuro.2008.02.005

[11] Mull, M., Nijenhuis, R.J., Backes, W.H., Krings, T. Wilmink, J.T. and Thron, A. (2007) Value and Limitations of Contrast-Enhanced MR Angiography in Spinal Arteriovenous Malformations and Dural Arteriovenous Fistulas. AJNR American Journal of Neuroradiology, 28, 1249-1258.http://dx.doi.org/10.3174/ajnr.A0612

[12] Luetmer, P.H., Lane, J.I., Gilbertson, J.R., Bernstein, M.A., Huston, J. $3^{\text {rd }}$ and Atkinson, J.L. (2005) Preangiographic Evaluation of Spinal Dural Arteriovenous Fistulas with Elliptic Cnetric Contrast-Enhanced MR Angiography and Effect on Radiation Dose and Volume of Iodinated Contrast Material. AJNR American Journal of Neuroradiology, 26, 711-718. 